Published in final edited form as:

Curr Infect Dis Rep. 2016 January ; 18(1): 2. doi:10.1007/s11908-015-0510-9.

\title{
Carbapenem-Resistant Enterobacteriaceae Infections in Children
}

\author{
Kathleen Chiotos, MD ${ }^{1,2,3}$, Jennifer H. Han, MD, MSCE ${ }^{4,5,6}$, and Pranita D. Tamma, MD, \\ MHS $^{7}$ \\ ${ }^{1}$ Division of Critical Care, Department of Anesthesia and Critical Care Medicine, The Children's \\ Hospital of Philadelphia, Philadelphia, PA \\ ${ }^{2}$ Division of Infectious Diseases, The Children's Hospital of Philadelphia, Philadelphia, PA \\ ${ }^{3}$ Center for Pediatric Clinical Effectiveness, Department of Pediatrics, The Children's Hospital of \\ Philadelphia, Philadelphia, PA \\ ${ }^{4}$ Division of Infectious Diseases, Department of Medicine, Perelman School of Medicine, \\ University of Pennsylvania, Philadelphia, PA \\ ${ }^{5}$ Center for Clinical Epidemiology and Biostatistics, Perelman School of Medicine, University of \\ Pennsylvania, Philadelphia, PA \\ ${ }^{6}$ Department of Biostatistics and Epidemiology, Perelman School of Medicine, University of \\ Pennsylvania, Philadelphia, PA \\ ${ }^{7}$ Division of Infectious Diseases, Department of Pediatrics, The Johns Hopkins University School \\ of Medicine, Baltimore, MD
}

\section{Abstract}

Carbapenem-resistant Enterobacteriaceae (CRE) are an emerging global public health threat. Infections due to CRE are associated with significant morbidity and mortality. Few therapeutic options are available for treatment of these infections, and optimal antibiotic treatment regimens are unclear. Along with the rapidly increasing prevalence of CRE in the United States and worldwide, several studies have described the epidemiology of CRE in the adult population. While CRE is now also reported sporadically in children, there is a significant lack of data on the epidemiology, risk factors, treatment, and outcomes in this population. This article provides a comprehensive review of what is known to date about CRE, including clinical and molecular epidemiology, microbiologic diagnosis, antibiotic treatment options, and outcomes. In particular, this review will focus on the available data on CRE in the pediatric population.

Corresponding author: Kathleen Chiotos, MD, Division of Infectious Diseases, Abramson Research Building, Room 1202, The Children's Hospital of Philadelphia, $34^{\text {th }}$ and Civic Center Blvd, Philadelphia, PA 19104, Tel: (215) 313-9399, Fax: (215) 590-2025, chiotosk@email.chop.edu.

Additional authors contact information:

Jennifer Han, MD, MSCE, Division of Infectious Diseases, Department of Medicine, Perelman School of Medicine, University of

Pennsylvania, 811 Blockley Hall, 423 Guardian Drive, Philadelphia, PA 19104, Tel: (215) 573-5439, jennifer.han@uphs.upenn.edu Pranita Tamma, MD, MHS, Division of Infectious Diseases, Department of Pediatrics, Johns Hopkins University School of Medicine, 200 North Wolfe Street, Suite 3149, Baltimore, MD 21287, Tel: (443) 287-4099, ptamma1@jhmi.edu

Potential conflicts of interest: None to report for all authors. 


\section{Keywords}

Carbapenem-resistant Enterobacteriaceae (CRE); carbapenemases; KPCs; pediatrics; gramnegative; multidrug-resistant organisms; resistant gram-negative bacteria

\section{Introduction}

Infections due to antibiotic-resistant gram-negative organisms, including those caused by extended spectrum beta-lactamase (ESBL)-producing Enterobacteriaceae, are increasing worldwide and represent a significant public health threat [1]. Carbapenems have historically been the most effective antimicrobial therapy for infections due to these organisms. However, over the past two decades, carbapenem-resistant Enterobacteriaceae (CRE) have increasingly been identified as causes of healthcare-associated infections in adult patients. Infections due to CRE in adult populations have been associated with poor clinical outcomes, including mortality rates as high as 40-65\% [2-5]. CRE have been reported infrequently in children, but increasing reports of infections both in the United States and worldwide, along with an increasingly medically complex pediatric population suggest that CRE may emerge as a significant nosocomial pathogen in pediatric centers over the coming years [6].

\section{Molecular Epidemiology of CRE}

The most common Enterobacteriaceae exhibiting carbapenem resistance is Klebsiella pneumoniae followed by Enterobacter species, with other members of the Enterobacteriaceae reported less frequently [7, 8]. Resistance to carbapenems develops by one of two general mechanisms: enzymatic or non-enzymatic mechanisms. The former involves production of carbapenemases, enzymes which hydrolyze the $\beta$-lactam ring of carbapenem antibiotics. The later includes production of ESBLs and/or AmpC cephalosporinases in conjunction with decreases in membrane permeability. Carbapenemase-producing Enterobacteriaceae have largely been responsible for the rapid worldwide spread of CRE owing to easily transferred mobile genetic elements (e.g., plasmids, transposons) that encode carbapenemase genes. In contrast, decreases in membrane permeability are frequently associated with a loss in fitness to the organism and reduced transmissibility $[6,9]$. It therefore becomes important for epidemiologic purposes to differentiate organisms that are carbapenemase producers (CP-CRE) from those that are carbapenem-resistant due to other mechanisms (non-CP-CRE). For example, while contact precautions are generally recommended for both CP-CRE and non-CP-CRE, CP-CRE may warrant more aggressive infection control interventions such as targeted active surveillance [10].

While the Ambler classification system differentiates carbapenemases based on molecular structure, key differences also exist with regard to specific resistance patterns, inhibitors, phenotypic characteristics, and geographic distributions (Table 1). Ambler class A carbapenemases include chromosomally as well as plasmid encoded carbapenemases, the most common of which is $K$. pneumoniae carbapenemase (KPC). The KPC gene bla $\mathrm{KPC}_{\mathrm{KC}}$ is associated with a mobile transposon Tn4401 which has become established in some strains 
particularly adept at clonal expansion, such as $K$. pneumoniae sequence type (ST) 258 .

ST258 is largely responsible for the epidemiologic success of KPC-producing organisms [9, 11]. KPC production accounts for up to $80 \%$ of carbapenem resistance in the United States and an increasing proportion of CRE worldwide [12-14]. While KPC producers have been identified on all continents, the United States, Israel, Greece, Poland, Italy, China, Columbia, Brazil, and Argentina represent highly endemic areas [15].

Ambler class B carbapenemases include the Verona integrin-encoded metallo-betalactamases (VIM), IMP types, and New Delhi metallo-beta-lactamases (NDM-1). VIM and IMP have historically been linked to the Mediterranean basin and Asia, although they have been isolated worldwide, including in the United States. NDM-1, which is endemic to the Indian subcontinent and accounts for as many as 50\% of CRE isolates in this region, has now been identified throughout Europe, parts of Asia and Africa, and in the United States, often in individuals who have traveled from endemic regions $[4,9]$.

Ambler class D carbapenemases are oxacillinases and most notably include the OXA-48 carbapenemases. Class D carbapenemases are most frequently identified in Acinetobacter species. However, the OXA-48 carbapenemase has been increasingly reported in $K$. pneumoniae and E. coli isolates. OXA-48 was originally associated with the Middle East, North Africa, and remain endemic in Turkey, but like other groups of carbapenemases, have been isolated in several other geographic regions worldwide, including the United States [9, 16]. As international migration and medical tourism continue to increase, it is anticipated that geographic distinctions for the various carbapenemase-encoding genes will eventually disappear.

\section{Epidemiology and Risk Factors}

Defining the extent of the global CRE epidemic has been challenging for a number of reasons, including changing definitions of carbapenem resistance in both the United States and Europe, technical challenges of distinguishing carbapenemase production from other mechanisms of resistance, and incomplete reporting of CRE isolates worldwide [4, 10, 1618]. In the United States, the Centers for Disease Control and Prevention (CDC) defines CRE as organisms resistant to imipenem, meropenem, doripenem, or ertapenem or documentation that isolates are carbapenemase-producing [19]. This revised 2015 definition is an attempt to increase the sensitivity of the prior definition which did not include ertapenem resistance, as a small subset of organisms that produce KPC are susceptible to all carbapenems except ertapenem. Additionally, the previous requirement for isolates to exhibit resistance to all third-generation cephalosporins was removed to simplify application of the definition and to account for some OXA-48 type carbapenemases that may not exhibit resistance to third-generation cephalosporins [19-21].

In the United States, KPC was first identified in a $K$. pneumoniae isolate in North Carolina in 2001 [22]. Since then, KPC-producing isolates have been identified in 48 states, The District of Columbia, and Puerto Rico, although rates remain highest in the mid-Atlantic region $[10,12]$. The CDC's National Healthcare Safety Network (NHSN) reported carbapenem resistance in approximately $12 \%$ of $K$. pneumoniae and approximately $2 \%$ of $E$. 
coli isolates in 2009-2010, with 3.9\% of acute care hospitals and $17.8 \%$ of long term acute care hospitals (LTACHs) reporting at least one healthcare-associated CRE infection [2, 7]. Other recent studies report overall carbapenem resistance rates between $3.4 \%$ to $6 \%$ in $K$. pneumoniae and $0.8 \%$ in E. coli, a significant increase in the past decade primarily due to spread of KPC $[8,12,23,24]$.

Risk factors for CRE infections in adult patients have been incompletely characterized and conflicting results are reported in various studies likely due to differences in study design, inclusion of patients with both CRE infection and colonization, and diversity of study populations and health systems with reports from both the developed and developing world. LTACH residence has been well established as a risk factor for CRE, with $30 \%$ of residents demonstrating colonization with CRE in a point prevalence study of Chicago area LTACHs $[2,25]$. Prior antibiotic exposure has also been shown to confer an increased risk of colonization and/or infection with CRE, although the agents implicated vary significantly among studies with anti-pseudomonal penicillins, beta-lactam/beta-lactamase inhibitors, extended-spectrum cephalosporins, carbapenems, glycopeptides and quinolones all reported [26-32]. Other identified risk factors include presence of medical devices, intensive care unit (ICU) stay, solid organ transplant, renal failure, and bedbound status [5, 27-32]. Reported mortality rates due to CRE infection among adult patients have varied across studies, but were as high as $65 \%$ in some populations despite antibiotic therapy $[3,5]$.

\section{Pediatric Patients}

Far less is known about CRE epidemiology and risk factors in children. Currently available data are largely limited to case reports, controlled and uncontrolled case series, and one systematic review summarizing available data through 2012 [6]. A recently published study reported that the frequency of carbapenem resistance increased from 0\% in 1999-2000 to 0.47\% in 2010-2011 among Enterobacteriaceae isolates in children in the United States [33]. Worldwide, the frequency of meropenem non-susceptibility among $K$. pneumoniae and E. coli isolates recovered from children is approximately $4 \%$ and $<1 \%$, respectively [34]. Most large national surveillance reports fail to include children's hospitals or do not report pediatric specific data $[7,8,12,23,24]$.

Available data, however, suggests that CRE epidemiology, risk factors, and outcomes in the pediatric population reflect many of the trends observed in studies in adult populations. Nosocomial outbreaks with CP-CRE have been reported in pediatric facilities, including a California neonatal intensive care unit (NICU), a Spanish pediatric intensive care unit (PICU), and a Nepali NICU associated with IMP, VIM, and NDM-1 carbapenemases, respectively [35-37]. Pediatric long-term care facilities (LTCF), similar to adult LTACHs, may also serve as reservoirs of CRE; in the one pediatric report investigating this association, KPC rectal colonization was detected in 12 of 26 (46\%) pediatric LTCF residents [38]. Lastly, geographic trends in the distribution of carbapenemases are generally comparable in adult and pediatric populations [6, 35-37, 39-51].

Patient-level risk factors for CRE infection in children include underlying chronic medical conditions, invasive medical devices, frequent or prolonged hospitalizations, prior antibiotic exposure, age, and travel from endemic regions [6, 35, 36, 39-44, 46, 48-50, 52, 53]. 
Neonates seem to be a particularly at risk group with several reported outbreaks of NDM-1producing Enterobacteriaceae in the neonatal population in the Indian subcontinent [35, 37, $47,51]$. Although mortality rates of children vary across reports, the highest rates are seen in the neonatal population [35-37, 39-49, 51].

In the United States, a recent case series in the pediatric population included $11 \mathrm{CRE}$ isolates from 10 patients, of which 4 had clinical infection with CRE in Los Angeles, California. Carbapenemases were identified in 5 isolates, including two NDM-1 type carbapenemases and three KPCs. Three patients had CRE isolated from the blood, 3 from the urine, and 2 each from wounds and the respiratory tract. All but one patient had underlying medical conditions, including 3 with malignancies. In addition, 8 patients had central venous catheters, 1 had traveled to India, and 8 had been treated at an adult hospital in the year prior to CRE diagnosis. In the 6 months prior to CRE isolation, patients received a median of 28 days of antibiotics. Four patients in this series died [39].

Another series from the United States described 6 patients with CRE infection including 5 with bacteremia and 1 with a urinary tract infection (UTI) in Seattle, Washington. One patient had KPC-producing $K$. pneumoniae, two had IMP-4-producing E. coli, one had SME-producing Serratia marcescens and two had ESBL-producing E. coli with associated porin mutations. Five of the 6 patients had underlying hematologic malignancies and the sixth had a renal transplant. There was one death [40].

Lastly, a series of 13 patients with CRE isolated from clinical specimens identified in The District of Columbia reported underlying medical conditions and prior antibiotic exposure in 12 of 13 patients, of which gastrointestinal disease was most common. When compared to age-matched controls, previous antibiotic use, including carbapenems, third-generation cephalosporins, and fluoroquinolones, was significantly associated with CRE isolation. Among these 13 patients, 7 developed CRE infection, including bacteremia (2), UTIs (3), skin and soft tissue (1), and intra-abdominal infection (1). No patients died in this series. Of note, this study did not distinguish CP-CRE from non-CP-CRE [52].

Internationally, Greece, Italy, the United Kingdom (UK), China, Pakistan, and Turkey have all described similarly small case series of CRE in children, with findings corroborating those from US studies [41-45, 50 53]. A report from Italy identified 15 CP-CRE isolates, of which 6 were associated with clinical infection, including 4 bacteremias and 2 UTIs. There were 2 deaths. In this study, 11 of the patients had malignancies and all patients were treated with $\geq 20$ days of gram-negative active antibiotics in the 90 days prior to CRE detection [41]. In the UK study, notable findings include detection of CRE colonization in approximately $5 \%$ of study patients. Of these, $21 \%$ developed an infection with the same organism, suggesting that active surveillance could be of potential utility in identifying patients at risk of subsequent CRE infection [45]. An even higher burden of CRE colonization was detected in a Turkish series with $29 \%$ of pediatric patients colonized with CRE. These patients were more likely to have received a carbapenem prior to CRE isolation and have been transferred from another healthcare facility. Notably, $3.4 \%$ of these colonized patients went on to develop CRE infections [53]. Another Turkish study reported that $84 \%$ of CP-CRE 
colonization occurred in pediatric patients, which was attributed to the ease of dissemination within open units common in neonatal and pediatric ICUs [46].

The epidemiology of CRE in the neonatal population warrants separate consideration as they are among the most vulnerable pediatric patients. In a Pakistani NICU, a reported $72 \%$ of $K$. pneumoniae isolates were carbapenem resistant [47]. Between 2007 and 2011, carbapenem resistance in an Indian NICU were reported as 33\% for E. coli and 13\% for K. pneumoniae, with $14 \%$ of Enterobacteriaceae producing NDM-1 [51]. Lastly, a large outbreak of NDM-1 producing Enterobacteriaceae was described in a Nepali NICU involving 26 neonates and was associated with a $64 \%$ mortality rate [37].

\section{Diagnosis of CRE}

In 2010, the Clinical and Laboratory Standards Institute (CLSI) lowered carbapenem susceptibility minimal inhibitory concentration (MIC) breakpoints for Enterobacteriaceae in part to eliminate the need for confirmatory testing for carbapenemase production. The reported MICs under these new guidelines reflect achievable pharmacokineticpharmacodynamic (PK-PD) targets with standard, intermittent carbapenem dosing, regardless of the mechanism of resistance [54]. The result was a decrease in the carbapenem MIC considered susceptible for imipenem and meropenem from $\leq 4 \mu \mathrm{g} / \mathrm{mL}$ to $\leq 1 \mu \mathrm{g} / \mathrm{mL}$. The MIC breakpoint for ertapenem was lowered from $\leq 2 \mu \mathrm{g} / \mathrm{mL}$ to $\$ 0.25 \mu \mathrm{g} / \mathrm{mL}$, and subsequently increased to $\leq 0.5 \mu \mathrm{g} / \mathrm{mL}$ [18]. These guidelines, along with the CDC's revised definition of CRE, remove the requirement for additional testing for carbapenemase production [55]. While this approach is appealing for its relative ease of interpretation and decreased need for laboratory resources, failure to differentiate CP-CRE from non-CP-CRE has important epidemiologic implications, including the potential for more aggressive infection control interventions than may be necessary for non-CP-CRE. An additional concern is that the lowered breakpoints may result in use of unnecessarily broad, toxic, or less effective antibiotic therapy, such as polymyxins or tigecycline $[56,57]$. Therefore, depending on considerations such as regional endemicity and laboratory resources, confirmatory testing for the presence of a carbapenemase may be warranted $[10,17,55,58]$.

\section{Phenotypic Testing}

The modified Hodge test (MHT), also known as the "clover leaf" test, is a phenotypic test for carbapenemase production in which the suspected carbapenemase-producing organism is streaked onto Mueller-Hinton agar. Carbapenemase production is detected when the isolate produces a carbapenemase, allowing for growth of a carbapenem-susceptible strain along the streak of the inoculum toward the carbapenem disc. The MHT is highly sensitive for class A and D carbapenemases, inexpensive, and easy to perform, and is included in CLSI and CDC recommendations for confirmatory testing for carbapenemase production [10, 18]. However, it is limited by frequent false positive results from ESBL or AmpC production combined with porin mutations, subjective interpretation, 72-96 hour turnaround time from the time of culture attainment, and reduced sensitivity in detecting MBL-type carbapenemases, although this is improved with addition of zinc to the medium [59, 60].

Curr Infect Dis Rep. Author manuscript; available in PMC 2017 January 01. 
Another group of phenotypic assays are the rapid chromogenic assays, most notably the Carba NP test in which a bacterial lysate is incubated with imipenem and a color change occurs in the presence of imipenem hydrolysis. Tazobactam or EDTA can also be added to samples to discriminate among Ambler class A, B, and D carbapenemases [61, 62]. Sensitivity of the Carba NP test varies among studies from $80 \%$ to $100 \%$, with lower sensitivity attributed to inconclusive results or false negatives with OXA-48 type carbapenemases, as well as for NDM-1-producing Proteus and Providencia isolates. Specificity remains $100 \%$ across studies, which when coupled with the ease of performance and rapid turn around time (30 minutes to 2 hours), makes it an attractive alternative to the MHT [61-64]. Another advantage of chromogenic assays, as opposed to the MHT, are their ability to identify carbapenemases in nonfermenters such as Acinetobacter spp. and Pseudomonas spp. and not just Enterobacteriaceae. Other available rapid chromogenic assays include the Rapid CARB Screen Kit (Rosco Diagnostica), the Rapid CARB Blue Kit (Rosco Diagnostica), and the RAPIDEC CARBA NP assay (bioMérieux) [65].

\section{Genotypic testing}

Single and multiplex polymerase chain reaction (PCR) assays and microarray assays are available for common carbapenemases, including KPC, VIM, IMP, NDM, and OXA-48, with sensitivity and specificity of these tests approaching 100\% [66-68]. Clear advantages of these molecular methods include identification of specific carbapenemase genes with superior sensitivity and specificity as well as rapid turn around time. Disadvantages include relative expense of these methods as well as need for specialized equipment and trained personnel. Furthermore, use of these assays at present requires in-house validation as none are approved by the United States Food and Drug Administration (FDA) or recommended by the CLSI. Molecular methods only have the ability to detect resistance genes included in their platform and cannot detect novel carbapenemase genes. Enhancements to existing matrix-assisted laser desorption ionization-time of flight (MALDI-TOF) mass spectrometry observed in the research setting may enable this technology to detect CP-CREs in diagnostic laboratories in upcoming years [69-70].

\section{Screening for CRE}

The CDC provides a laboratory protocol for detection of CRE from rectal or perirectal swabs for surveillance purposes [71]. This process, which involves inoculation of the specimen onto MacConkey agar and use of carbapenem discs, has been validated for E. coli and Klebsiella species. Alternatively, chromogenic media are available commercially and have reasonable sensitivity for detection of CRE, are relatively inexpensive, and are easily interpretable [72, 73]. PCR-based methods are more sensitive than chromogenic media, but are associated with the same disadvantages described above [74].

\section{Treatment of Infections due to CRE}

Data on optimal treatment regimens for CRE are hampered by a lack of randomized trials evaluating the effectiveness of various therapeutic approaches. Current recommendations are guided by case reports, case series, and cohort studies with significant heterogeneity in populations and treatment regimens evaluated [75]. Overall, available data support 
combination therapy with at least 2 agents given significant reductions in mortality with this approach compared to monotherapy, with cited mortality rates as low as $13 \%[13,76-78]$. There are insufficient data at this point, however, to recommend a particular combination regimen, and antibiotic selection should be guided by clinical source of infection, local susceptibility patterns, and expert consultation.

\section{Carbapenems}

Currently available data suggest that carbapenem-containing combination therapy appears to have a mortality benefit over monotherapy or non-carbapenem containing combination therapy [13, 76-78]. Along these lines, the largest review to date evaluating treatment of CRE infections reported a mortality rate of $19 \%$ with carbapenem-containing combinations compared to $31 \%$ for carbapenem-sparing combinations [13]. Modeling studies suggest that prolonged or continuous infusion of meropenem can achieve target concentrations for isolates with MICs as high as $8 \mu \mathrm{g} / \mathrm{mL}-16 \mu \mathrm{g} / \mathrm{mL}$, although extrapolating this into clinical practice is difficult given a recent study reporting increased mortality with MICs between 2$8 \mu \mathrm{g} / \mathrm{mL}$ compared with MIC $\leq 1 \mu \mathrm{g} / \mathrm{mL}[79,80]$. Few data exist in the pediatric population on the potential benefit of extended or continuous infusions of carbapenems. However, carbapenems remain a mainstay of pediatric therapy based on the available adult literature and experience with these agents in pediatric populations [81, 82].

\section{Polymyxins}

Polymyxins, including colistin and polymyxin B, have re-emerged as first-line therapy for serious infections due to carbapenem-resistant organisms. However, their use is limited by increasing resistance rates, which have been reported to be as high as $25 \%$ for CRE and $8 \%$ for all Enterobacteriaceae [75, 83, 84]. In addition, polymyxins have been associated with significant renal toxicity, with rates of acute kidney injury occurring in as many as 40 to $60 \%$ of adult patients $[57,85]$. Available evidence suggests that mortality is higher in patients with CRE infections that are treated with colistin monotherapy compared with those receiving colistin-containing combination therapy $[77,86]$. No pediatric studies have directly evaluated the use of colistin for CRE specifically, but studies including patients with all multidrug-resistant gram-negative infections cite favorable outcomes in $70-80 \%$ of all patients, with incidence rates of acute kidney injury up to 20\% [87-89].

\section{Tigecycline}

Tigecycline remains active in vitro against the majority of Enterobacteriaceae in the United States, with resistance rates reported at $1.7 \%$ overall and less than $0.1 \%$ among pediatric patients. Among CRE, tigecycline resistance is reported in approximately $12 \%$ of isolates $[34,84,90]$. As with carbapenems and colistin, outcomes are poor with tigecycline monotherapy but improved when tigecycline is combined with another agent [76, 78, 91]. However, there are emerging data suggesting increased mortality in patients treated with tigecycline for any indication, the etiology of which is not well understood but thought to be most likely related to poorly sustained serum concentrations; as a result, the FDA has issued a black box warning for this agent $[56,92]$. In pediatric settings, experience with tigecycline is limited to case reports, and along with the potential for increased mortality, association 
with enamel hypoplasia, tooth discoloration, and availability of other agents should limit its use to situations when other treatment options have been exhausted [40, 41, 48, 49, 81, 93].

\section{Fosfomycin}

Fosfomycin remains active against most CRE isolates tested, and the intravenous form has been used successfully to treat CRE infections in adult patients outside the United States, generally in combination with a second agent due to the risk of developing resistance while on therapy $[94,95]$. Intravenous dosing is available for pediatric patients, although the intravenous form of the drug is not currently available in the United States [96]. The oral form of the drug, however, is available in the United States and achieves high concentrations in the urine, making it a favorable option for treatment of cystitis caused by CRE [48, 95].

\section{Emerging agents}

Ceftazidime-avibactam was approved in by the FDA in February 2015 following a priority review of phase II trial data showing efficacy and safety comparable to imipenem-cilastatin and meropenem for treatment of complicated urinary tract and intra-abdominal infections in adult patients [97, 98]. Avibactam is active against both KPC and some OXA-type carbapenemases but not metallo-beta-lactamases $[99,100]$. A phase I trial in pediatric patients was completed in late 2014 (NCT01893346) and two phase II studies enrolling infants as young as 3 months of age are currently ongoing to evaluate the role of ceftazidime-avibactam in children with complicated urinary-tract and intra-abdominal infections (NCT02475733 and NCT02497781) [101-103]. Other agents in development include ceftaroline-avibactam, imipenem - MK7655, biapenem - RPX7009, plazomicin (a novel aminoglycoside), and eravacycline, all with variable activity against the different carbapenemase classes based on existing in vitro data [75, 81, 104].

\section{Prevention and Control of CRE}

In the United States, the CDC recommends several "core measures" for CRE prevention and control, including hand hygiene, antimicrobial stewardship, minimizing use of invasive devices, and contact precautions and patient cohorting for patients with CRE colonization or infection [10, 16]. Surveillance procedures for CRE colonization in institutions with few or no CRE cases may include a point prevalence screen of those at highest risk, including LTACH residents, patients transferred from other facilities with known CRE transmission, patients who recently received medical care in regions of the world endemic for CRE, or patients admitted to high risk units such as ICUs [10]. If a patient harboring CRE is identified, institutions should screen epidemiologically linked contacts and units. In institutions with ongoing CRE transmission, active surveillance should be performed on admission in high-risk patients as well as periodically throughout their stay. Although outbreaks of carbapenemase-producing organisms have been difficult to control, there are data that if systematically implemented, rigorous infection control procedures can halt the spread of these organisms in both hospital and LTACH settings [105-107]. 


\section{Conclusion}

While CRE represents an emerging threat in pediatric patients, little data exist at the present time on epidemiology, risk factors, treatment, or outcomes in this increasingly vulnerable population. Furthermore, the lack of currently available treatment options is exacerbated by limited data on their use in pediatric patients, emerging resistance to these therapies, and a lack of clinical trials evaluating novel agents or PK-PD of many available CRE-active antibiotics in the pediatric population. Future research efforts should focus on filling these gaps in knowledge.

\section{Acknowledgments}

Financial support: This work was supported by the National Institutes of Health (T32HD060550 to K.C. and K01AI103028 to J.H.H.) and the Sherrilyn and Ken Fisher Center for Environmental Infectious Diseases, Division of Infectious Diseases of The Johns Hopkins University School of Medicine

Role of the funding agency: The funding agency had no role in the design and conduct of the study; collection, management, analysis, and interpretation of the data; or preparation, review, or approval of the manuscript.

\section{References}

$\square$ Of importance

\section{Of major importance}

1. Spellberg B, Guidos R, Gilbert D, Bradley J, Boucher HW, Scheld WM, et al. The epidemic of antibiotic-resistant infections: a call to action for the medical community from the Infectious Diseases Society of America. Clin Infect Dis. 2008; 46(2):155-164. [PubMed: 18171244]

2. Vital signs: carbapenem-resistant Enterobacteriaceae. MMWR. 2013; 62(9):165-170. [PubMed: 23466435]

3. Borer A, Saidel-Odes L, Riesenberg K, Eskira S, Peled N, Nativ R, et al. Attributable mortality rate for carbapenem-resistant Klebsiella pneumoniae bacteremia. Infect Control Hosp Epidemiol. 2009; 30(10):972-976. [PubMed: 19712030]

4. Gupta N, Limbago BM, Patel JB, Kallen AJ. Carbapenem-resistant Enterobacteriaceae: epidemiology and prevention. Clin Infect Dis. 2011; 53(1):60-67. [PubMed: 21653305]

5. Satlin MJ, Jenkins SG, Walsh TJ. The global challenge of carbapenem-resistant Enterobacteriaceae in transplant recipients and patients with hematologic malignancies. Clin Infect Dis. 2014; 58(9): 1274-1283. [PubMed: 24463280]

6. Logan LK. Carbapenem-resistant enterobacteriaceae: an emerging problem in children. Clin Infect Dis. 2012; 55(6):852-859. [PubMed: 22700827] Summarizes available pediatric reports of CRE infection through 2012, including epidemiology, risk factors, antimicrobial susceptibility, and treatment of these patients. This is the only review of pediatric CRE infection.

7. Sievert DMP, Ricks PP, Edwards JRMS, Schneider AMPH, Patel JP, Srinivasan AMD, et al Antimicrobial-resistant pathogens associated with healthcare-associated infections: summary of data reported to the National Healthcare Safety Network at the Centers for Disease Control and Prevention, 2009-2010. Infect Control Hosp Epidemiol. 2013; 34(1):1-14. [PubMed: 23221186]

8. Rhomberg PR, Jones RN. Summary trends for the Meropenem Yearly Susceptibility Test Information Collection Program: a 10-year experience in the United States (1999-2008). Diagn Microbiol Infect Dis. 2009; 65(4):414-426. [PubMed: 19833471]

9. Nordmann P, Naas T, Poirel L. Global spread of carbapenemase-producing Enterobacteriaceae. Emerg Infect Dis. 2011; 17(10):1791-1798. [PubMed: 22000347]

10. [Accessed 6 August 2015] Centers for Disease Control and Prevention. Carbapenem-resistant Enterobacteriaceae (CRE) Infection. 2015. http://www.cdc.gov/hai/organisms/cre/creclinicians.html 
11. Kitchel B, Rasheed JK, Patel JB, Srinivasan A, Navon-Venezia S, Carmeli Y, et al. Molecular epidemiology of KPC-producing Klebsiella pneumoniae isolates in the United States: clonal expansion of multilocus sequence type 258. Antimicrob Agents Chemother. 2009; 53(8):33653370. [PubMed: 19506063]

12. Kaiser RM, Castanheira M, Jones RN, Tenover F, Lynfield R. Trends in Klebsiella pneumoniae carbapenemase-positive K. pneumoniae in US hospitals: report from the 2007-2009 SENTRY Antimicrobial Surveillance Program. Diagn Microbiol Infect Dis. 2013; 76(3):356-360. [PubMed: 23659829]

13. Tzouvelekis LS, Markogiannakis A, Piperaki E, Souli M, Daikos GL. Treating infections caused by carbapenemase-producing Enterobacteriaceae. Clin Microbiol Infec. 2014; 20:862-872. [PubMed: 24890393] This is the largest and most recent review evaluating available literature on treatment of CRE infections in adult patients.

14. Guh AY, Bulens SN, Mu Y, Jacob JT, Reno J, Scott J, et al. Epidemiology of CarbapenemResistant Enterobacteriaceae in 7 US Communities, 2012-2013. JAMA. 2015; 314(14):14791487. [PubMed: 26436831]

15. Munoz-Price LS, Poirel L, Bonomo RA, Schwaber MJ, Daikos GL, Cormican M, et al. Clinical epidemiology of the global expansion of Klebsiella pneumoniae carbapenemases. Lancet Infect Dis. 2013; 13(9):785-796. [PubMed: 23969216]

16. Temkin E, Adler A, Lerner A, Carmeli Y. Carbapenem-resistant Enterobacteriaceae: biology, epidemiology, and management. Ann NY Acad Sci. 2014; 1323:22-42. [PubMed: 25195939] Recent review article on CRE in adult patients with emphasis on epidemiology and control.

17. Nordmann P, Poirel L. Strategies for identification of carbapenemase-producing Enterobacteriaceae. J Antimicrob Chemother. 2013; 68(3):487-489. [PubMed: 23104494]

18. Clinical and Laboratory Standards Institute. Performance standards for antimicrobial susceptibility testing. M100-S25. Wayne, PA: CLSI; 2015.

19. [Accessed 11 August 2015] Centers for Disease Control and Prevention. FAQs about Choosing and Implementing a CRE Definition. 2015. http://www.cdc.gov/hai/organisms/cre/definition.html

20. Landman D, Salamera J, Singh M, Quale J. Accuracy of carbapenem nonsusceptibility for identification of KPC-possessing Enterobacteriaceae by use of the revised CLSI breakpoints. J Clin Microbiol. 2011; 49(11):3931-3933. [PubMed: 21880962]

21. Chea N, Bulens SN, Kongphet-Tran T, Lynfield R, Shaw KM, Vagnone PS, et al. Improved phenotype-based definition for identifying carbapenemase producers among carbapenem-resistant Enterobacteriaceae. Emerg Infect Dis. 2015; 21(9):1611-1616. [PubMed: 26290955]

22. Yigit H, Queenan AM, Anderson GJ, Domenech-Sanchez A, Biddle JW, Steward CD, et al. Novel carbapenem-hydrolyzing beta-lactamase, KPC-1, from a carbapenem-resistant strain of Klebsiella pneumoniae. Antimicrob Agents Chemother. 2001; 45(4):1151-1161. [PubMed: 11257029]

23. Braykov NP, Eber MR, Klein EY, Morgan DJ, Laxminarayan R. Trends in resistance to carbapenems and third-generation cephalosporins among clinical isolates of Klebsiella pneumoniae in the United States, 1999-2010. Infect Control Hosp Epidemiol. 2013; 34(3):259_ 268. [PubMed: 23388360]

24. Davies TA, Marie Queenan A, Morrow BJ, Shang W, Amsler K, He W, et al. Longitudinal survey of carbapenem resistance and resistance mechanisms in Enterobacteriaceae and non-fermenters from the USA in 2007-09. J Antimicrob Chemother. 2011; 66(10):2298-2307. [PubMed: 21775338]

25. Lin MY, Lyles-Banks RD, Lolans K, Hines DW, Spear JB, Petrak R, et al. The importance of longterm acute care hospitals in the regional epidemiology of Klebsiella pneumoniae carbapenemaseproducing Enterobacteriaceae. Clin Infect Dis. 2013; 57(9):1246-1252. [PubMed: 23946222]

26. Kritsotakis EI, Tsioutis C, Roumbelaki M, Christidou A, Gikas A. Antibiotic use and the risk of carbapenem-resistant extended-spectrum-\{beta\}-lactamase-producing Klebsiella pneumoniae infection in hospitalized patients: results of a double case-control study. J Antimicrob Chemother. 2011; 66(6):1383-1391. [PubMed: 21454344]

27. Falagas ME, Rafailidis PI, Kofteridis D, Virtzili S, Chelvatzoglou FC, Papaioannou V, et al. Risk factors of carbapenem-resistant Klebsiella pneumoniae infections: a matched case control study. J Antimicrob Chemother. 2007; 60(5):1124-1130. [PubMed: 17884829] 
28. Wu D, Cai J, Liu J. Risk factors for the acquisition of nosocomial infection with carbapenemresistant Klebsiella pneumoniae. South Med J. 2011; 104(2):106-110. [PubMed: 21258230]

29. Gasink LB, Edelstein PH, Lautenbach E, Synnestvedt M, Fishman NO. Risk factors and clinical impact of Klebsiella pneumoniae carbapenemase-producing K. pneumoniae. Infect Control Hosp Epidemiol. 2009; 30(12):1180-1185. [PubMed: 19860564]

30. Swaminathan M, Sharma S, Poliansky Blash S, Patel G, Banach DB, Phillips M, et al. Prevalence and risk factors for acquisition of carbapenem-resistant Enterobacteriaceae in the setting of endemicity. Infect Control Hosp Epidemiol. 2013; 34(8):809-817. [PubMed: 23838221]

31. Schwaber MJ, Klarfeld-Lidji S, Navon-Venezia S, Schwartz D, Leavitt A, Carmeli Y. Predictors of carbapenem-resistant Klebsiella pneumoniae acquisition among hospitalized adults and effect of acquisition on mortality. Antimicrob Agents Chemother. 2008; 52(3):1028-1033. [PubMed: 18086836]

32. Patel G, Huprikar S, Factor SH, Jenkins SG, Calfee DP. Outcomes of carbapenem-resistant Klebsiella pneumoniae infection and the impact of antimicrobial and adjunctive therapies. Infect Control Hosp Epidemiol. 2008; 29(12):1099-1106. [PubMed: 18973455]

33. Logan LK, Renschler JP, Gandra S, Weinstein RA, Laxminarayan R. Centers for Disease Control and Prevention Epicenters Program. Carbapenem-resistant Enterobacteriaceae in children, United States, 1999-2012. Emerg Infect Dis. 2015 Nov. [Epub ahead of print.].

34. Kehl SC, Dowzicky MJ. Global assessment of antimicrobial susceptibility among Gram-negative organisms collected from pediatric patients between 2004 and 2012: results from the Tigecycline Evaluation and Surveillance Trial. J Clin Microbiol. 2015; 53(4):1286-1293. [PubMed: 25653413]

35. Limbago BM, Rasheed JK, Anderson KF, Zhu W, Kitchel B, Watz N, et al. IMP-producing carbapenem-resistant Klebsiella pneumoniae in the United States. J Clin Microbiol. 2011; 49(12): 4239-4245. [PubMed: 21998425]

36. Oteo J, Hernandez-Almaraz JL, Gil-Anton J, Vindel A, Fernandez S, Bautista V, et al. Outbreak of vim-1-carbapenemase-producing Enterobacter cloacae in a pediatric intensive care unit. Pediatr Infect Dis J. 2010; 29(12):1144-1146. [PubMed: 20686438]

37. Stoesser N, Giess A, Batty EM, Sheppard AE, Walker AS, Wilson DJ, et al. Genome sequencing of an extended series of NDM-producing Klebsiella pneumoniae isolates from neonatal infections in a Nepali hospital characterizes the extent of community- versus hospital-associated transmission in an endemic setting. Antimicrob Agents Chemother. 2014; 58(12):7347-7357. [PubMed: 25267672]

38. Viau RA, Hujer AM, Marshall SH, Perez F, Hujer KM, Briceno DF, et al. "Silent" dissemination of Klebsiella pneumoniae isolates bearing K. pneumoniae carbapenemase in a long-term care facility for children and young adults in Northeast Ohio. Clin Infect Dis. 2012; 54(9):1314-1321. [PubMed: 22492318]

39. Pannaraj PS, Bard JD, Cerini C, Weissman SJ. Pediatric carbapenem-resistant Enterobacteriaceae in Los Angeles, California, a high-prevalence region in the United States. Pediatr Infect Dis J. 2015; 34(1):11-16. [PubMed: 25093977] Largest and most recent series of pediatric CRE in the United States with information on frequency of carbapenemase production, risk factors, and outcomes. The clinical course of the 5 patients with carbapenemase-producing organisms are summarized in detail.

40. Little ML, Qin X, Zerr DM, Weissman SJ. Molecular diversity in mechanisms of carbapenem resistance in paediatric Enterobacteriaceae. Int J Antimicrob Ag. 2012; 39(1):52-57. A second series of pediatric CRE in the United States which contains information on specific carbapenemases detected as well as a detailed clinical course for each patient.

41. Colombo S, Scolfaro C, Calitri C, Denina M, Carraro F, De Intinis G, et al. Carbapenemaseproducing enterobacteriaceae (CPE) in the pediatric setting: results from an 18-month survey. Infect Control Hosp Epidemiol. 2014; 35(5):599-601. [PubMed: 24709738] Description of epidemiology, risk factors, and outcomes for 6 Italian patients with carbapenemase-producing Enterobacteriaceae infection.

42. Cendejas E, Gomez-Gil R, Gomez-Sanchez P, Mingorance J. Detection and characterization of Enterobacteriaceae producing metallo-beta-lactamases in a tertiary-care hospital in Spain. Clin Microbiol Infec. 2010; 16(2):181-183. [PubMed: 19624502] 
43. Maltezou HC, Kontopidou F, Katerelos P, Daikos G, Roilides E, Theodoridou M. Infections caused by carbapenem-resistant Gram-negative pathogens in hospitalized children. Pediatr Infect Dis J. 2013; 32(4):e151-e154. [PubMed: 23249908]

44. Liu Y, Li XY, Wan LG, Jiang WY, Li FQ, Yang JH. Molecular characterization of the bla(KPC-2) gene in clinical isolates of carbapenem-resistant Klebsiella pneumoniae from the pediatric wards of a Chinese hospital. Can J Microbiol. 2012; 58(10):1167-1173. [PubMed: 22978676]

45. Drew RJ, Turton JF, Hill RL, Livermore DM, Woodford N, Paulus S, et al. Emergence of carbapenem-resistant Enterobacteriaceae in a UK paediatric hospital. J Hosp Infect. 2013; 84(4): 300-304. [PubMed: 23831281]

46. Alp E, Percin D, Colakoglu S, Durmaz S, Kurkcu CA, Ekincioglu P, et al. Molecular characterization of carbapenem-resistant Klebsiella pneumoniae in a tertiary university hospital in Turkey. J Hosp Infect. 2013; 84(2):178-180. [PubMed: 23623803]

47. Saleem AF, Qamar FN, Shahzad H, Qadir M, Zaidi AK. Trends in antibiotic susceptibility and incidence of late-onset Klebsiella pneumoniae neonatal sepsis over a six-year period in a neonatal intensive care unit in Karachi, Pakistan. Int J Infect Dis. 2013; 17(11):e961-e965. [PubMed: 23759260]

48. Green DA, Srinivas N, Watz N, Tenover FC, Amieva M, Banaei N. A pediatric case of New Delhi metallo-beta-lactamase-1-producing Enterobacteriaceae in the United States. Pediatr Infect Dis J. 2013; 32(11):1291-1294. [PubMed: 23743543]

49. Dara JS, Chen L, Levi MH, Kreiswirth BN, Pellett Madan R. Microbiological and genetic characterization of carbapenem-resistant isolates from pediatric patients. J Pediatric Infect Dis Soc. 2014; 3(1):e10-e14. [PubMed: 24567846]

50. Qamar MU, Nahid F, Walsh TR, Kamran R, Zahra R. Prevalence and clinical burden of NDM-1 positive infections in pediatric and neonatal patients in Pakistan. Pediatr Infect Dis J. 2015; 34(4): 452-454. [PubMed: 25764102]

51. Datta S, Roy S, Chatterjee S, Saha A, Sen B, Pal T, et al. A five-year experience of carbapenem resistance in Enterobacteriaceae causing neonatal septicaemia: predominance of NDM-1. PloS one. 2014; 9(11):e112101. [PubMed: 25406074] Reports on a recent CRE outbreak in an Indian NICU involving a large number of neonates. Risk factors for CRE infection and mortality are discussed.

52. Dirajlal-Fargo S, DeBiasi R, Campos J, Song X. Carbapenem-resistant Enterobacteriaceae in pediatric patients: epidemiology and risk factors. Infect Control Hosp Epidemiol. 2014; 35(4): 447-449. [PubMed: 24602957] Large series describing clinical characteristics of patients with CRE. The authors also performed a case control study showing increased risk of CRE colonization with prior antibiotic exposure.

53. Ulu-Kilic A, Alp E, Percin D, Cevahir F, Altay-Kurkcu C, Ozturk A, et al. Risk factors for carbapenem resistant Klebsiella pneumoniae rectal colonization in pediatric units. J Infect Dev Ctries. 2014; 8(10):1361-1364. [PubMed: 25313618] Describes frequency of and risk factors for rectal colonization with CRE in pediatric patients.

54. Dudley MN, Ambrose PG, Bhavnani SM, Craig WA, Ferraro MJ, Jones RN. Background and rationale for revised clinical and laboratory standards institute interpretive criteria (Breakpoints) for Enterobacteriaceae and Pseudomonas aeruginosa: I. Cephalosporins and Aztreonam. Clin Infect Dis. 2013; 56(9):1301-1309. [PubMed: 23334813]

55. Doern CD, Dunne WM Jr, Burnham CA. Detection of Klebsiella pneumoniae carbapenemase (KPC) production in non-Klebsiella pneumoniae Enterobacteriaceae isolates by use of the Phoenix, Vitek 2, and disk diffusion methods. J Clin Microbiol. 2011; 49(3):1143-1147. [PubMed: 21209164]

56. Yahav D, Lador A, Paul M, Leibovici L. Efficacy and safety of tigecycline: a systematic review and meta-analysis. J Antimicrob Chemother. 2011; 66(9):1963-1971. [PubMed: 21685488]

57. Garonzik SM, Li J, Thamlikitkul V, Paterson DL, Shoham S, Jacob J, et al. Population pharmacokinetics of colistin methanesulfonate and formed colistin in critically ill patients from a multicenter study provide dosing suggestions for various categories of patients. Antimicrob Agents Chemother. 2011; 55(7):3284-3294. [PubMed: 21555763]

58. Hrabak J, Chudackova E, Papagiannitsis CC. Detection of carbapenemases in Enterobacteriaceae: a challenge for diagnostic microbiological laboratories. Clin Microbiol Infec. 2014; 20(9):839-853. 
[PubMed: 24813781] Concise review of methods for laboratory detection of CRE and carbapenemase production.

59. Girlich D, Poirel L, Nordmann P. Value of the modified Hodge test for detection of emerging carbapenemases in Enterobacteriaceae. J Clin Microbiol. 2012; 50(2):477-479. [PubMed: 22116154]

60. Carvalhaes CG, Picao RC, Nicoletti AG, Xavier DE, Gales AC. Cloverleaf test (modified Hodge test) for detecting carbapenemase production in Klebsiella pneumoniae: be aware of false positive results. J Antimicrob Chemother. 2010; 65(2):249-251. [PubMed: 19996141]

61. Nordmann P, Poirel L, Dortet L. Rapid detection of carbapenemase-producing Enterobacteriaceae. Emerg Infect Dis. 2012; 18(9):1503-1507. [PubMed: 22932472]

62. Dortet L, Poirel L, Nordmann P. Rapid identification of carbapenemase types in Enterobacteriaceae and Pseudomonas spp. by using a biochemical test. Antimicrob Agents Chemother. 2012; 56(12): 6437-6440. [PubMed: 23070158]

63. Vasoo S, Cunningham SA, Kohner PC, Simner PJ, Mandrekar JN, Lolans K, et al. Comparison of a novel, rapid chromogenic biochemical assay, the Carba NP test, with the modified Hodge test for detection of carbapenemase-producing Gram-negative bacilli. J Clin Microbiol. 2013; 51(9):30973101. [PubMed: 23824767]

64. Tijet N, Boyd D, Patel SN, Mulvey MR, Melano RG. Evaluation of the Carba NP test for rapid detection of carbapenemase-producing Enterobacteriaceae and Pseudomonas aeruginosa. Antimicrob Agents Chemother. 2013; 57(9):4578-4580. [PubMed: 23817380]

65. Dortet L, Agathine A, Naas T, Cuzon G, Poirel L, Nordmann P. Evaluation of the RAPIDEC(R) CARBA NP, the Rapid CARB Screen(R) and the Carba NP test for biochemical detection of carbapenemase-producing Enterobacteriaceae. J Antimicrob Chemother. 2015 [Advanced access 9 August 2015]

66. van der Zee A, Roorda L, Bosman G, Fluit AC, Hermans M, Smits PH, et al. Multi-centre evaluation of real-time multiplex PCR for detection of carbapenemase genes OXA-48, VIM, IMP, NDM and KPC. BMC Infect Dis. 2014; 14:27. [PubMed: 24422880]

67. Stuart JC, Voets G, Scharringa J, Fluit AC, Leverstein-Van Hall MA. Detection of carbapenemaseproducing Enterobacteriaceae with a commercial DNA microarray. J Med Microbiol. 2012; 61(Pt 6):809-812. [PubMed: 22383444]

68. Naas T, Cuzon G, Bogaerts P, Glupczynski Y, Nordmann P. Evaluation of a DNA microarray (Check-MDR CT102) for rapid detection of TEM, SHV, and CTX-M extended-spectrum betalactamases and of KPC, OXA-48, VIM, IMP, and NDM-1 carbapenemases. J Clin Microbiol. 2011; 49(4):1608-1613. [PubMed: 21325547]

69. Hrabak J, Studentova V, Walkova R, Zemlickova H, Jakubu V, Chudackova E, et al. Detection of NDM-1, VIM-1, KPC, OXA-48, and OXA-162 carbapenemases by matrix-assisted laser desorption ionization-time of flight mass spectrometry. J Clin Microbiol. 2012; 50(7):2441-2443. [PubMed: 22553235]

70. Lasserre C, De Saint Martin L, Cuzon G, Bogaerts P, Lamar E, Glupczynski Y, et al. Efficient detection of carbapenemase activity in Enterobacteriaceae by matrix-assisted laser desorption ionization-time of flight mass spectrometry in less than 30 minutes. J Clin Microbiol. 2015; 53(7): 2163-2171. [PubMed: 25926485]

71. [Accessed 8 August 2015] Centers for Disease Control and Prevention. Laboratory Protocol for Detection of Carbapenem-Resistant or Carbapenemase-Producing, Klebsiella spp. and E. coli from Rectal Swabs. 2015. http://www.cdc.gov/HAI/pdfs/labSettings/Klebsiella_or_Ecoli.pdf

72. Vasoo S, Lolans K, Li H, Prabaker K, Hayden MK. Comparison of the CHROMagar KPC, Remel Spectra CRE, a direct ertapenem disk method for the detection of KPC-producing Enterobacteriaceae from perirectal swabs. Diagn Microbiol Infect Dis. 2014; 78(4):356-359. [PubMed: 24439449]

73. Adler A, Navon-Venezia S, Moran-Gilad J, Marcos E, Schwartz D, Carmeli Y. Laboratory and clinical evaluation of screening agar plates for detection of carbapenem-resistant Enterobacteriaceae from surveillance rectal swabs. J Clin Microbiol. 2011; 49(6):2239-2242. [PubMed: 21471338] 
74. Singh K, Mangold KA, Wyant K, Schora DM, Voss B, Kaul KL, et al. Rectal screening for Klebsiella pneumoniae carbapenemases: comparison of real-time PCR and culture using two selective screening agar plates. J Clin Microbiol. 2012; 50(8):2596-2600. [PubMed: 22622443]

75. Morrill HJ, Pogue JM, Kaye KS, LaPlante KL. Treatment options for carbapenem-resistant Enterobacteriaceae infections. Open Forum Infect Dis. 2015; 2(2):ofv050. [PubMed: 26125030] The most recent review synthesizing available literature for various treatment options for CRE infections in adult patients.

76. Daikos GL, Tsaousi S, Tzouvelekis LS, Anyfantis I, Psichogiou M, Argyropoulou A, et al. Carbapenemase-producing Klebsiella pneumoniae bloodstream infections: lowering mortality by antibiotic combination schemes and the role of carbapenems. Antimicrob Agents Chemother. 2014; 58(4):2322-2328. [PubMed: 24514083]

77. Qureshi ZA, Paterson DL, Potoski BA, Kilayko MC, Sandovsky G, Sordillo E, et al. Treatment outcome of bacteremia due to KPC-producing Klebsiella pneumoniae: superiority of combination antimicrobial regimens. Antimicrob Agents Chemother. 2012; 56(4):2108-2113. [PubMed: 22252816]

78. Tumbarello M, Viale P, Viscoli C, Trecarichi EM, Tumietto F, Marchese A, et al. Predictors of mortality in bloodstream infections caused by Klebsiella pneumoniae carbapenemase-producing K. pneumoniae: importance of combination therapy. Clin Infect Dis. 2012; 55(7):943-950. [PubMed: 22752516]

79. Roberts JA, Kirkpatrick CM, Roberts MS, Robertson TA, Dalley AJ, Lipman J. Meropenem dosing in critically ill patients with sepsis and without renal dysfunction: intermittent bolus versus continuous administration? Monte Carlo dosing simulations and subcutaneous tissue distribution. J Antimicrob Chemother. 2009; 64(1):142-150. [PubMed: 19398460]

80. Patel TS, Nagel JL. Clinical outcomes of Enterobacteriaceae infections stratified by carbapenem MICs. J Clin Microbiol. 2015; 53(1):201-205. [PubMed: 25378572]

81. Hsu AJ, Tamma PD. Treatment of multidrug-resistant Gram-negative infections in children. Clin Infect Dis. 2014; 58(10):1439-1448. [PubMed: 24501388] This article summarizes available pediatric data for treatment of CRE infections.

82. Tamma PD, Jenh AM, Milstone AM. Prolonged beta-lactam infusion for Gram-negative Infections. Pediatr Infect Dis J. 2011; 30(4):336-337. [PubMed: 21407037]

83. Petrosillo N, Giannella M, Lewis R, Viale P. Treatment of carbapenem-resistant Klebsiella pneumoniae: the state of the art. Expert Rev Anti Infect Ther. 2013; 11(2):159-177. [PubMed: 23409822]

84. Sader HS, Castanheira M, Flamm RK, Mendes RE, Farrell DJ, Jones RN. Tigecycline activity tested against carbapenem-resistant Enterobacteriaceae from 18 European nations: results from the SENTRY surveillance program (2010-2013). Diagn Microbiol Infect Dis. 2015 Jun 23. [Epub ahead of print].

85. Akajagbor DS, Wilson SL, Shere-Wolfe KD, Dakum P, Charurat ME, Gilliam BL. Higher incidence of acute kidney injury with intravenous colistimethate sodium compared with polymyxin B in critically ill patients at a tertiary care medical center. Clin Infect Dis. 2013; 57(9):1300-1303. [PubMed: 23840000]

86. Balkan II, Aygun G, Aydin S, Mutcali SI, Kara Z, Kuskucu M, et al. Blood stream infections due to OXA-48-like carbapenemase-producing Enterobacteriaceae: treatment and survival. Int J Infect Dis. 2014; 26:51-56. [PubMed: 24998423]

87. Tamma PD, Newland JG, Pannaraj PS, Metjian TA, Banerjee R, Gerber JS, et al. The use of intravenous colistin among children in the United States: results from a multicenter, case series. Pediatr Infect Dis J. 2013; 32(1):17-22. [PubMed: 22935871]

88. Kapoor K, Jajoo M, Dublish S, Dabas V, Gupta S, Manchanda V. Intravenous colistin for multidrug-resistant gram-negative infections in critically ill pediatric patients. Pediatr Crit Care Med. 2013; 14(6):e268-e272. [PubMed: 23689704]

89. Paksu MS, Paksu S, Karadag A, Sensoy G, Asilioglu N, Yildizdas D, et al. Old agent, new experience: colistin use in the paediatric Intensive Care Unit--a multicentre study. Int J Antimicrob Ag. 2012; 40(2):140-144. 
90. Sader HS, Farrell DJ, Flamm RK, Jones RN. Variation in potency and spectrum of tigecycline activity against bacterial strains from U.S. medical centers since its approval for clinical use (2006 to 2012). Antimicrob Agents Chemother. 2014; 58(4):2274-2280. [PubMed: 24492361]

91. Sbrana F, Malacarne P, Viaggi B, Costanzo S, Leonetti P, Leonildi A, et al. Carbapenem-sparing antibiotic regimens for infections caused by Klebsiella pneumoniae carbapenemase-producing K. pneumoniae in intensive care unit. Clin Infect Dis. 2013; 56(5):697-700. [PubMed: 23155147]

92. Kaewpoowat Q, Ostrosky-Zeichner L. Tigecycline : a critical safety review. Expert Opin Drug Saf. 2015; 14(2):335-342. [PubMed: 25539800]

93. Purdy J, Jouve S, Yan JL, Balter I, Dartois N, Cooper CA, et al. Pharmacokinetics and safety profile of tigecycline in children aged 8 to 11 years with selected serious infections: a multicenter, open-label, ascending-dose study. Clin Ther. 2012; 34(2):496.e1-507.e1. [PubMed: 22249106]

94. Michalopoulos A, Virtzili S, Rafailidis P, Chalevelakis G, Damala M, Falagas ME. Intravenous fosfomycin for the treatment of nosocomial infections caused by carbapenem-resistant Klebsiella pneumoniae in critically ill patients: a prospective evaluation. Clin Microbiol Infec. 2010; 16(2): 184-186. [PubMed: 19694767]

95. Karageorgopoulos DE, Wang R, Yu XH, Falagas ME. Fosfomycin: evaluation of the published evidence on the emergence of antimicrobial resistance in Gram-negative pathogens. J Antimicrob Chemother. 2012; 67(2):255-268. [PubMed: 22096042]

96. Traunmuller F, Popovic M, Konz KH, Vavken P, Leithner A, Joukhadar C. A reappraisal of current dosing strategies for intravenous fosfomycin in children and neonates. Clin Pharmacokinet. 2011; 50(8):493-503. [PubMed: 21740073]

97. Lucasti C, Popescu I, Ramesh MK, Lipka J, Sable C. Comparative study of the efficacy and safety of ceftazidime/avibactam plus metronidazole versus meropenem in the treatment of complicated intra-abdominal infections in hospitalized adults: results of a randomized, double-blind, Phase II trial. J Antimicrob Chemother. 2013; 68(5):1183-1192. [PubMed: 23391714]

98. Vazquez JA, Gonzalez Patzan LD, Stricklin D, Duttaroy DD, Kreidly Z, Lipka J, et al. Efficacy and safety of ceftazidime-avibactam versus imipenem-cilastatin in the treatment of complicated urinary tract infections, including acute pyelonephritis, in hospitalized adults: results of a prospective, investigator-blinded, randomized study. Curr Med Res Opin. 2012; 28(12):19211931. [PubMed: 23145859]

99. Castanheira M, Mills JC, Costello SE, Jones RN, Sader HS. Ceftazidime-avibactam activity tested against Enterobacteriaceae isolates from U.S. hospitals (2011 to 2013) and characterization of beta-lactamase-producing strains. Antimicrob Agents Chemother. 2015; 59(6):3509-3517. [PubMed: 25845862]

100. Castanheira M, Farrell SE, Krause KM, Jones RN, Sader HS. Contemporary diversity of betalactamases among Enterobacteriaceae in the nine U.S. census regions and ceftazidime-avibactam activity tested against isolates producing the most prevalent beta-lactamase groups. Antimicrob Agents Chemother. 2014; 58(2):833-838. [PubMed: 24247134]

101. [Accessed 14 August 2015] Safety and Tolerability of Ceftazidime-Avibactam for Pediatric Patients With Suspected or Confirmed Infections. 2015. https://clinicaltrials.gov/ct2/show/ NCT01893346

102. [Accessed 20 October 2015] Evaluation of Safety, Pharmacokinetics and Efficacy of CAZ-AVI With Metronidazole in Childern Aged 3 Months to 18 Years Old With Complicated Intraabdominal Infections (cIAIs). 2015. https://clinicaltrials.gov/ct2/show/NCT02475733

103. [Accessed 20 October 2015] Evaluation of Safety, Pharmacokinetics and Efficacy of Ceftazidime and Avibactam (CAZ-AVI) Compared With Cefepime in Children From 3 Months to Less Than 18 Years of Age With Complicated Urinary Tract Infections (cUTIs). 2015. https:// clinicaltrials.gov/ct2/show/NCT02497781

104. Boucher HW, Talbot GH, Benjamin DK Jr, Bradley J, Guidos RJ, Jones RN, et al. 10 × '20 Progress--development of new drugs active against gram-negative bacilli: an update from the Infectious Diseases Society of America. Clin Infect Dis. 2013; 56(12):1685-1694. [PubMed: 23599308]

105. Schwaber MJ, Lev B, Israeli A, Solter E, Smollan G, Rubinovitch B, et al. Containment of a country-wide outbreak of carbapenem-resistant Klebsiella pneumoniae in Israeli hospitals via a nationally implemented intervention. Clin Infect Dis. 2011; 52(7):848-855. [PubMed: 21317398] 
106. Kochar S, Sheard T, Sharma R, Hui A, Tolentino E, Allen G, et al. Success of an infection control program to reduce the spread of carbapenem-resistant Klebsiella pneumoniae. Infect Control Hosp Epidemiol. 2009; 30(5):447-452. [PubMed: 19301985]

107. Munoz-Price LS, Hayden MK, Lolans K, Won S, Calvert K, Lin M, et al. Successful control of an outbreak of Klebsiella pneumoniae carbapenemase-producing K. pneumoniae at a long-term acute care hospital. Infect Control Hosp Epidemiol. 2010; 31(4):341-347. [PubMed: 20175685] 


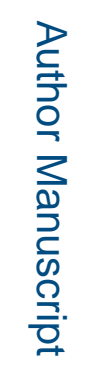

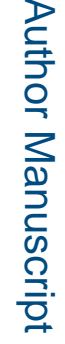

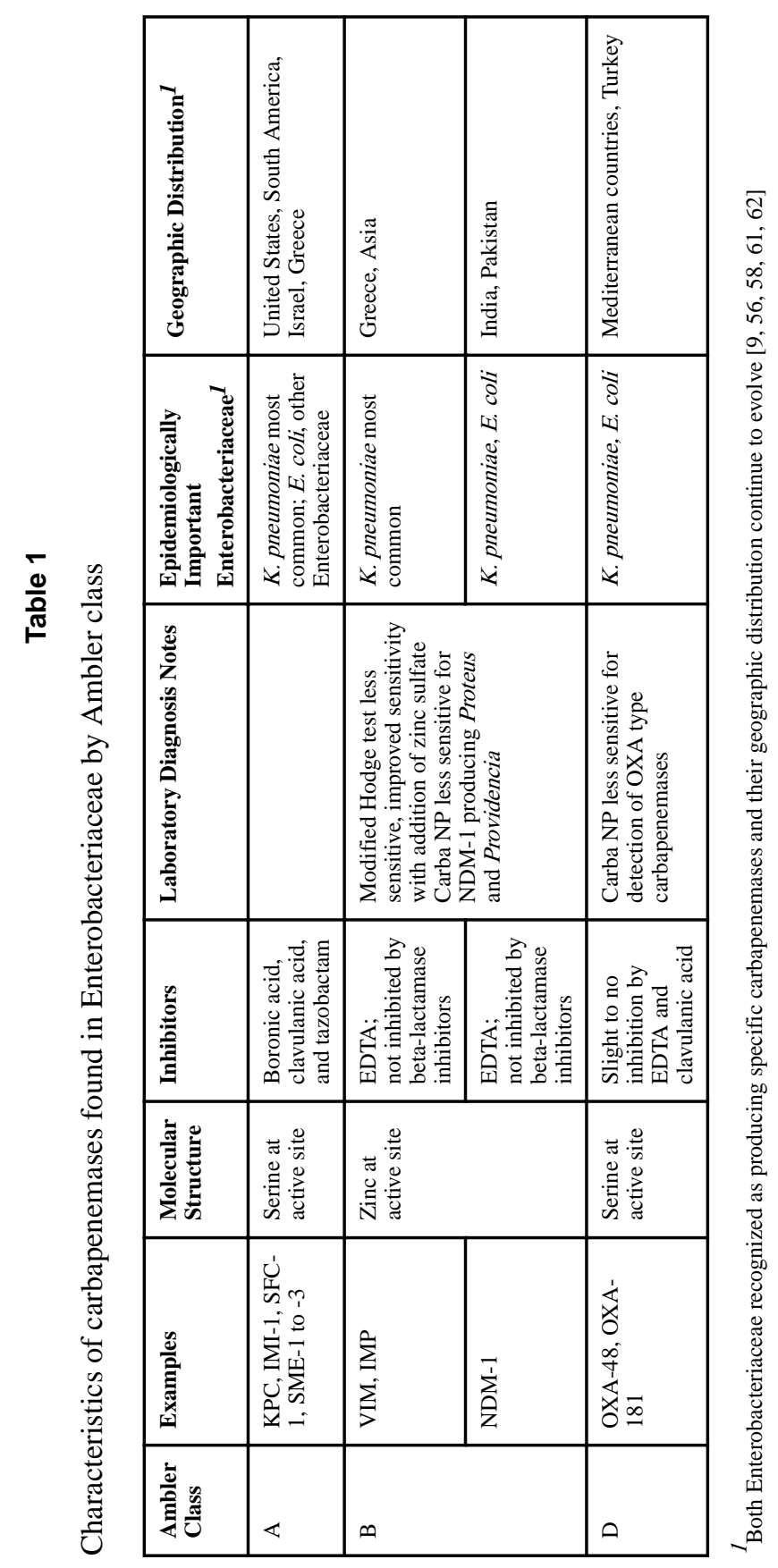

Curr Infect Dis Rep. Author manuscript; available in PMC 2017 January 01. 